Sādhanā Vol. 40, Part 2, April 2015, pp. 549-575. (C) Indian Academy of Sciences

\title{
Analysis of double support phase of biped robot and multi-objective optimization using genetic algorithm and particle swarm optimization algorithm
}

\author{
REGA RAJENDRA and DILIP KUMAR PRATIHAR*
}

Soft Computing Laboratory, Mechanical Engineering Department, Indian Institute of Technology, Kharagpur, Kharagpur 721 302, India

e-mail: dkpra@mech.iitkgp.ernet.in

MS received 16 September 2013; revised 7 November 2014; accepted 21 November 2014

\begin{abstract}
This paper deals with multi-objective optimization in gait planning of a 7-dof biped robot during its double support phase, while ascending and descending some staircases. For determining dynamic balance margin of the robot in terms of zero-moment point, its double support phase has been assumed to be consisting of two single support phases on non-coincidental parallel surfaces. Thus, dynamic balance margin of the biped robot during its double support phase is obtained by using a virtual zero-moment point of the system. Moreover, a smooth transition from single to double support phases in a cycle is to be maintained for the walking robots. Two contrasting objectives, namely power consumption and dynamic balance margin have been considered during optimization. Pareto-optimal fronts of solutions are obtained using genetic algorithm and particle swarm optimization algorithm, separately. To the best of the authors' knowledge, it is the first attempt to solve multi-objective optimization problem in double support phase of a biped robot.
\end{abstract}

Keywords. Optimal gait planning; genetic algorithm; particle swarm optimization.

\section{Introduction}

Biped robots are extensively studied by researchers. A biped robot should be able to walk on various terrains, such as flat, sloping surfaces, staircases and others, as the situation demands.

A biped robot's walking cycle consists of single support phase (SSP) and double support phase (DSP). It is in the SSP during the major portion of its walking cycle, and only a small fraction of the cycle time is utilized in the DSP. Hence, both the phases of walking are needed to be studied in detail to arrive at a complete knowledge of the biped walking systems on different terrains. An extensive study has been conducted on biped walking in the SSP for different terrains, namely flat surface, sloping surface, staircase and others, using different approaches. Most of the studies

*For correspondence 
used the concepts of centre of mass (COM), centre of gravity (COG), zero-moment point (ZMP) (Vukobratovic et al 1970), or the physics of inverted pendulum to dynamically balance the biped robot walking on different terrains. Although the double support is an important phase of the walking cycle, it has received less attention in terms of research. Only some limited studies have been conducted on the DSP of biped robots till date. This paper concentrates on analysis of the double support phase of a biped walking through some staircases.

A considerable amount of work had been carried out in the past related to mechanical design, development and testing of biped robots through computer simulations and/or real experiments. Hemami \& Farnsworth (1997) studied postural and gait stability of a planar five-link model of the biped robot in its DSP after considering both open and closed loops, and solved it by using d'Alembert's principle of motions. Simulations were conducted on the nonlinear system with linear feedbacks and satisfactory results had been obtained. Li et al (1991) proposed that if the preset ZMP of the trunk was measured, and control was achieved using a set of joint motion patterns, then stable walking could have been obtained by the biped robot for the walking cycle (both SSP and DSP). They experimentally verified the same on a biped robot named WL-12RIII. They used force-moment sensors to calculate the ZMP in SSP for both the feet on flat surface walking. The system ZMP was calculated off-line by considering the ZMP of each foot for the DSP. It is to be noted that there was a variation between the preset and measured values of ZMP of the system. Mitobe et al (1997) discussed the control problem of a biped walking robot during its DSP. The biped robot's DSP motion was considered as that of a manipulator under holonomic constraints. The trunk's COG position was controlled to track a desired trajectory for stability with respect to the world frame. This approach could achieve efficient walking. The control scheme was successfully applied to an experimental walking robot, and the results demonstrated the effectiveness of the method. Ito \& Kawasaki (2000) focussed on maintaining stability by controlling the ZMP in-between the two support regions of a two-link mechanism in a sagittal plane. To maintain the balance of the robot, the ground reactions forces should be positive. Hence, control laws for the ground reaction were proposed and verified through computer simulations. Wieber (2002) predicted that as long as the projection of mass of a robot walking on flat surface could lie within the convex hull of the support region, the stability of walking system could be guaranteed. This stability criterion was tested through numerical analysis.

Jamshidi \& Rostami (2008) exploited the pure dynamic synthesis of multi-body systems with sophisticated kinematics to study the motion of the robot during its DSP motion using Pontryagin's maximum principle. The closed chain DSP was considered as an open one at specific joint, and penalty method was used to locate the leg at prescribed location. A feasible set of motions was taken into consideration by using inequality constraints to limit the joint motion. Ground reaction force components were also used as control variables. The proposed technique could generate optimal free motions. The proposal was implemented for solving a numerical simulation using two-point boundary value problems using a shooting method, which required a less number of parameters to characterize the gait. Ito et al (2008) had studied side-to-side walking of biped robot in DSP in a lateral plane, where the variation of environment and ZMP were kept constant. This method was applied in the in-place stepping motion and stability of the method was examined both analytically as well as through computer simulations. Finally, the effectiveness of this method was demonstrated on an experiment using real robot. Sardain \& Bessonnet (2004) tried to solve the problem in DSP motion for the implementation of fast and dynamic walking gaits (for example, anthropomorphic gaits), especially on uneven terrain. For flat or uneven terrain, the authors analytically proved the coincidence of centre of pressure (COP) and ZMP for dynamic stable walking. But, for the non-coincidental or parallel planes, 
they proposed the existence of virtual ZMP for dynamic stable walking and the same was proved by solving numerical examples related to walking on some staircases.

A few studies dealt with intelligent control of biped robots also, some of those studies are discussed here. Lin et al (2010) developed a fuzzy logic (FL)-based technique combined with modern control theory for dynamic stabilization and locomotion control of biped motion. The performance of fuzzy control rules was evaluated for the biped walking on horizontal plane surface and climbing up a flight of stairs. Experimental results confirmed the effectiveness and applicability of the proposed fuzzy stabilization tuning approach. Vundavilli et al (2007a, b), Vundavilli \& Pratihar (2010, 2011a, b) used soft computing-based approaches to study dynamically balanced gaits of a 7-dof biped robot in its SSP for different terrains, namely staircase, sloping surface and ditches. However, their study focussed on optimization of a single objective in a cycle. The present study is an extension of the above work, where more realistic conditions for dynamic balancing in the DSP of the biped robot have been considered. It has been posed as a multi-objective optimization problem and solved using a genetic algorithm (GA) and particle swarm optimization (PSO) algorithm, separately.

Most of the studies on biped robots, available in the literature, are related to their walking on flat surface. However, a biped robot should be able to walk on rough terrains also, such as staircases, and others. The problems related to locomotion of biped robots on rough terrains are more complex (from the analysis and control points of view) compared to those on flat surface. Rough terrain locomotion of biped robots has not yet received enough attention, till date. In some of the above studies, gait planning tasks of biped robots had been formulated as single objective optimization problems. However, there is a scope to study multi-objective optimization in gait planning of biped robots particularly during its DSP, where at least two conflicting objectives may be considered to obtain Pareto-optimal front of solutions.

In the present work, an attempt has been made to formulate gait planning problem of a 7dof biped robot in its DSP by considering two separate SSPs. During each SSP, the robot is assumed to be a serial manipulator consisting of four links. The ZMP is determined for each SSP. Then, both the SSPs are combined to form a DSP. The biped robot will have to generate dynamically balanced gaits, while walking through the staircases after consuming minimum power. This problem related to the DSP of the biped robot has been posed as a multi-objective optimization one. Two conflicting objectives, such as minimization of power consumption and maximization of dynamic balance margin have been considered in the present study. A GA and PSO algorithm have been utilized to yield Pareto-optimal front of solutions separately. A comparison on the performances of these two optimization algorithms has also been presented.

The remaining part of this paper has been organized as follows: Section 2 deals with mathematical formulation of the problem. Section 3 explains the proposed algorithms to solve the said problems. Results are stated and discussed in section 4. Some concluding remarks are made in section 5 .

\section{Mathematical formulation of the problem}

The present study deals with an analysis of a 7-dof (that is, three at hip, two at knee and two at ankles) biped robot ascending and descending some staircases. It consists of two ankles, two lower legs, two upper legs and a trunk connected through seven rotary joints (Vundavilli et al 2007a, b). 


\subsection{Staircase ascending}

The biped walking cycle consists of two phases: SSP (that is, when one foot is in contact with the ground and the other is in air) and DSP (that is, when both the feet are in contact with the ground as shown in figure 1).

Here, $L_{1}, \ldots, L_{7}$ are lengths of the links and $m_{1}, \ldots, m_{7}$ are their lumped masses; $r_{1}, \ldots, r_{7}$ represent the locations of the respective lumped masses. Moreover, $x_{1}, x_{2}$ denote the locations of feet placement on the ground. The joint angles $\left(\theta_{2}, \ldots, \theta_{6}\right)$ are assumed to be positive, if these are measured in the anti-clockwise sense with respect to the vertical axis. Moreover, both the angles: $\theta_{1}$ and $\theta_{7}$ are assumed to be equal to zero. Here, $s_{w}$ and $s_{h}$ indicate the width and height of the staircase, respectively; and $X_{1}$ and $X_{2}$ denote the projected point of the trunk mass on the double support region. It is important to mention that the motion of the robot has been considered in the sagittal plane only. Figure 1 shows 2-D model of a biped robot having 7 dof only. The fourth link is massless, and it indicates hip, where three joints are assumed to be coinciding.

In the present work, DSP of the biped robot has been modelled as two SSPs. The robot consisting of seven links is represented as two serial manipulators having four links each. The mass of each link is assumed to be concentrated at a point lying on it. For simplicity, the movement of the biped robot and its balance are considered in one direction only. During the DSP, both the feet are on the ground carrying the total load of the robot. For continuity of walking, the information at the end of SSP is used at the beginning of DSP, in terms of joint angles: $\theta_{2}, \theta_{3}$, trunk angle $\theta_{4}$, position of the hip joint $\left(l_{1}, h_{1}\right)$ (refer to figure 1 ). It is to be noted that the trunk mass $m_{4}$ has been distributed into two parts, such as $m_{41}$ and $m_{42}$ (as shown in figure 2), according to its position with respect to two supporting feet (refer to figure 1 and Eqs. (1) and (2)).

$$
\begin{aligned}
& m_{41}=\frac{m_{4} X_{2}}{X_{1}+X_{2}}, \\
& m_{42}=\frac{m_{4} X_{1}}{X_{1}+X_{2}} .
\end{aligned}
$$

For simplicity, the hip trajectory (in Cartesian coordinate system) is assumed to follow a straight line having a slope equal to that of the staircase to ensure repeatability conditions of the cycles. A small hip movement $(\delta l, \delta h)$ in $\mathrm{X}, \mathrm{Z}$ directions are fed as inputs to the biped for the DSP motion to find the system dynamic stability in $\mathrm{X}$ direction. From figure $1, h_{1}, l_{1}$ can be determined as follows:

$$
\begin{gathered}
h_{1}=L_{2} \cos \theta_{2}+L_{3} \cos \theta_{3}, \\
l_{1}=L_{2} \sin \theta_{2}+L_{3} \sin \theta_{3} .
\end{gathered}
$$

By squaring and adding Eqs. (3) and (4), we get $\theta_{2}-\theta_{3}=\cos ^{-1}\left(\left(h_{1}^{2}+l_{1}^{2}-L_{2}^{2}-L_{3}^{2}\right) / 2 L_{2} L_{3}\right)=\phi_{1}$. Now, $h_{1}, l_{1}$ can be written in terms of $\phi_{1}$, as given below.

$$
\begin{aligned}
& h_{1}=\left(L_{2}+L_{3} \cos \phi_{1}\right) \cos \theta_{2}+\left(L_{3} \sin \phi_{1}\right) \sin \theta_{2} \\
& l_{1}=\left(L_{2}+L_{3} \cos \phi_{1}\right) \sin \theta_{2}-\left(L_{3} \sin \phi_{1}\right) \cos \theta_{2} .
\end{aligned}
$$




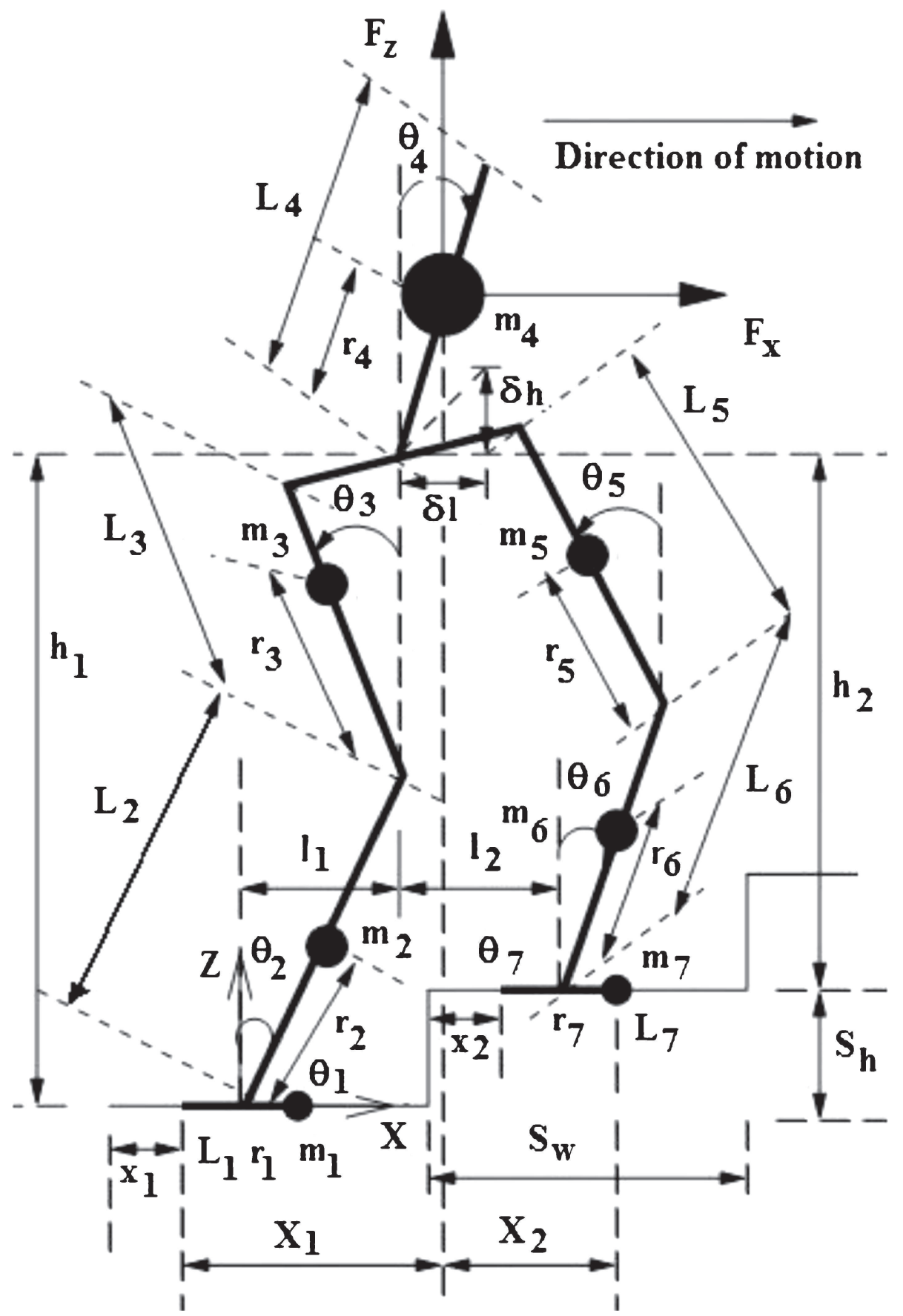

Figure 1. A schematic view of a biped robot (in its DSP) ascending the staircase. (assumption: Three joints coincide at the hip). 


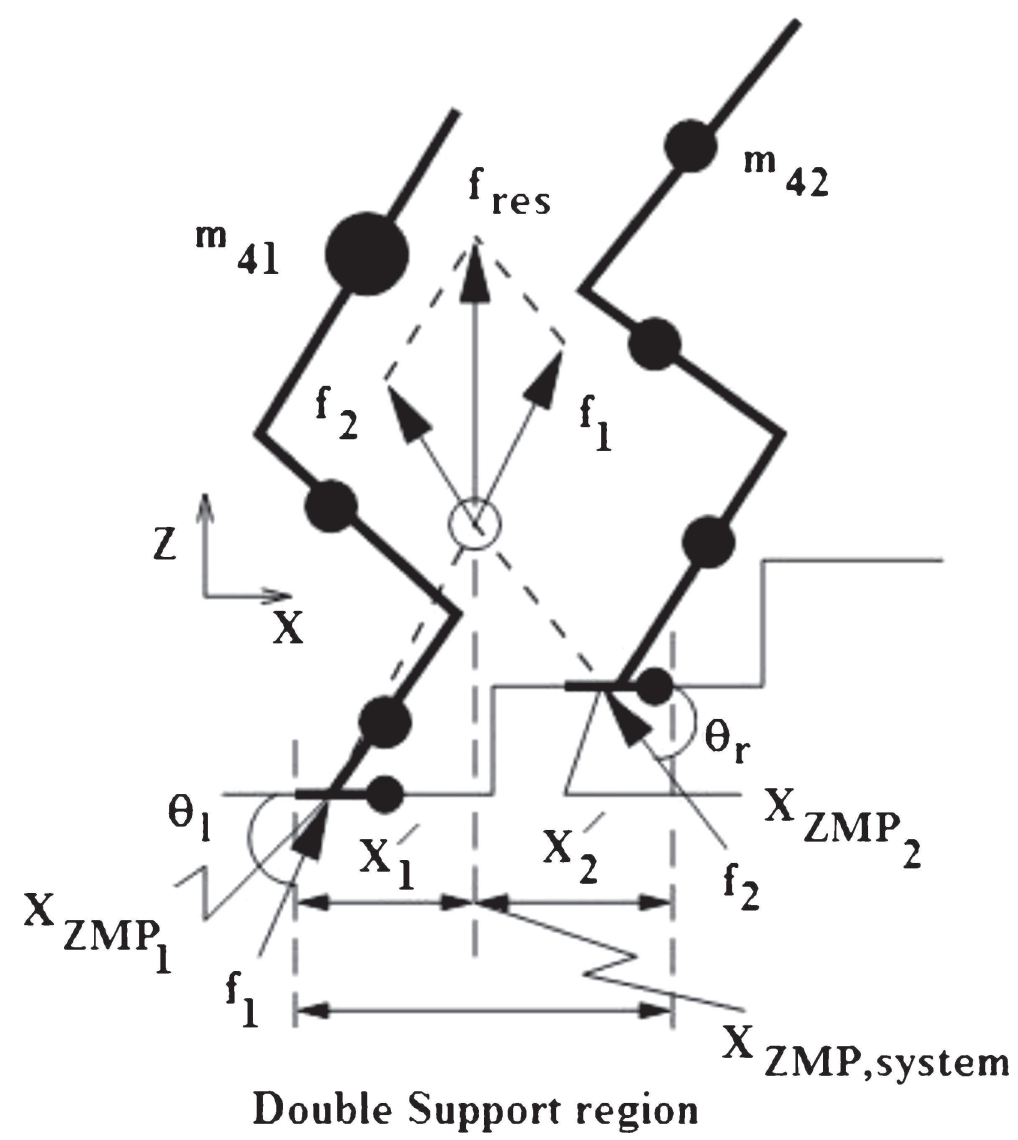

Figure 2. Calculation of system ZMP in double support phase in the sagittal view.

Now, by multiplying Eq. (5) by $l_{3} \sin \phi_{1}$ and Eq. (6) by $\left(L_{2}+L_{3} \cos \phi_{1}\right)$, and then adding, we get the joint angle $\theta_{2}$ in SSP of the robot, as follows:

$$
\theta_{2}=\sin ^{-1}\left(\frac{h_{1} L_{3} \sin \varphi_{1}+l_{1}\left(L_{2}+L_{3} \cos \varphi_{1}\right)}{\left(L_{2}+L_{3} \cos \varphi_{1}\right)^{2}+\left(L_{3} \sin \varphi_{1}\right)^{2}}\right),
$$

Thus, $\theta_{3}$ can be calculated as $\theta_{3}=\theta_{2}-\phi_{1}$. Similarly, the angles: $\theta_{5}$ and $\theta_{6}$ can be determined using the information of hip movement height $\left(h_{2}\right)$ and distance of the supporting ankle from the projection of hip joint $\left(l_{2}\right)$ is as follows:

$$
\theta_{6}=\sin ^{-1}\left(\frac{h_{2} L_{5} \sin \varphi_{2}+l_{2}\left(L_{6}+L_{5} \cos \varphi_{2}\right)}{\left(L_{6}+L_{5} \cos \varphi_{2}\right)^{2}+\left(L_{5} \sin \varphi_{2}\right)^{2}}\right),
$$

where $L_{5}$ and $L_{6}$ are the lengths of the links: $\varphi_{2}=\cos ^{-1}\left(\left(h_{2}^{2}+l_{2}^{2}-L_{6}^{2}-L_{5}^{2}\right) / 2 L_{5} L_{6}\right)$. The angle: $\theta_{5}$ can be obtained using the expression: $\theta_{5}=\theta_{6}-\varphi_{2}$.

A cycle has been divided into three equal time intervals for the purpose of analysis. The friction between the foot and the ground is assumed to be sufficient enough to prevent the slipping. Moreover, the generated gaits are to be dynamically balanced. For a dynamically balanced gait, 
the ZMP should lie inside the foot support polygon region. The robot is said to be dynamically balanced, when the ZMP lies inside the foot support polygon. The robot is checked for its dynamic balance using the concept of ZMP. As mentioned earlier, the DSP of the robot has been assumed to be consisting of two SSPs, for the ease of analysis. During the SSP of each ground foot, the ZMP is determined separately, using Eq. (9). The problem of DSP has been solved by combining these two SSPs, as shown in figure 2.

$$
x_{Z M P_{j}}=\frac{\sum_{i=1}^{4}\left(I_{i} \dot{\omega}_{i}+m_{i} x_{i}\left(\ddot{z}_{i}-g\right)-m_{i} \ddot{x}_{i} z_{i}\right)}{\sum_{i=1}^{4} m_{i}\left(\ddot{z}_{i}-g\right)},
$$

where $j=1,2 ; I_{i}$ denotes the moment of inertia of $i^{t h}$ link $\left(\mathrm{kg}-\mathrm{m}^{2}\right) ; \dot{\omega}_{i}$ is the angular acceleration of $i^{t h}$ link in $\left(\mathrm{rad} / \mathrm{s}^{2}\right) ; m_{i}$ denotes the mass of $i^{t h}$ link ( $\mathrm{kg}$ ) (for $i=4, m_{i}$ is to be replaced by $\left.m_{i j}\right) ;\left(x_{i}, z_{i}\right)$ is the coordinate of $i^{t h}$ lumped mass; $g$ is the acceleration due to gravity $\left(\mathrm{m} / \mathrm{s}^{2}\right)$, $\ddot{z}_{i}$ represents the acceleration of $i^{t h}$ link in $z$-direction $\left(\mathrm{m} / \mathrm{s}^{2}\right) ; \ddot{x}_{i}$ is the acceleration of $i^{t h}$ link in $x$-direction $\left(\mathrm{m} / \mathrm{s}^{2}\right)$.

Torque $\tau$ required at each joint of the robot for its locomotion has been determined using Lagrange formulation ( $\mathrm{Fu}$ et al 1987). Figure 3 shows the D-H parameters setting at various joints of the robot. The relationship between the generalized parameters $\left(q_{i}\right)$ and joint angles $\left(\theta_{i}\right)$ are as follows:

$$
\begin{aligned}
& q_{1}=\theta_{1} ; q_{2}=\left(90-\theta_{2}\right) ; q_{3}=\left(\theta_{2}-\theta_{3}\right) ; q_{4}=\left(\theta_{3}-\theta_{4}\right) \\
& q_{5}=\left(\theta_{4}-\theta_{5}\right) ; q_{6}=\left(\theta_{5}-\theta_{6}\right) ; q_{7}=\left(\theta_{7}-\left(90-\theta_{6}\right)\right) .
\end{aligned}
$$

The generalized angles are assumed to follow cubic polynomials in order to ensure their smooth variations, as given below

$$
q_{i}(t)=a_{i 0}+a_{i 1} t+a_{i 2} t^{2}+a_{i 3} t^{3},
$$

where $i=1,2, \ldots, n$ joints, and $a_{i 0}, a_{i 1}, a_{i 2}, a_{i 3}$ are the coefficients, whose values are to be determined using some known conditions. The angular velocity and acceleration can be determined by differentiating $q_{i}(t)$ with respect to time once and twice, respectively.

The amount of power consumed by $i^{\text {th }}$ joint can be calculated as the product of motor torque $\tau$ (refer to the Appendix A) and angular velocity. If the amount of heat loss of the motor Nishii et al (1998) is considered, the average power consumption over a cycle of time period $T$ is calculated as follows:

$$
P_{i}=\frac{1}{T} \sum_{i=1}^{n} \int_{0}^{T}\left(\left|\tau_{i} \dot{q}_{i}\right|+K \tau_{i}^{2}\right) d t
$$

where $\mathrm{K}$ is a constant, whose value has been assumed to be equal to 0.025 (Nishii et al 1998); $\mathrm{n}$ indicates the number of joints.

The ground reaction forces are formulated based on the assumption that the contact tips of the feet with the ground can be modelled as hard point contacts with friction, which indicates that interaction between the tip of the leg and ground is limited to three components of force: one normal and two tangential to the surface. The components of ground reaction forces for the first and second supporting legs are denoted by $f_{1}=\left[f_{x_{1}}, f_{y_{1}}, f_{z_{1}}\right]^{T}$ and $f_{2}=\left[f_{x_{2}}, f_{y_{2}}, f_{z_{2}}\right]^{T}$, 


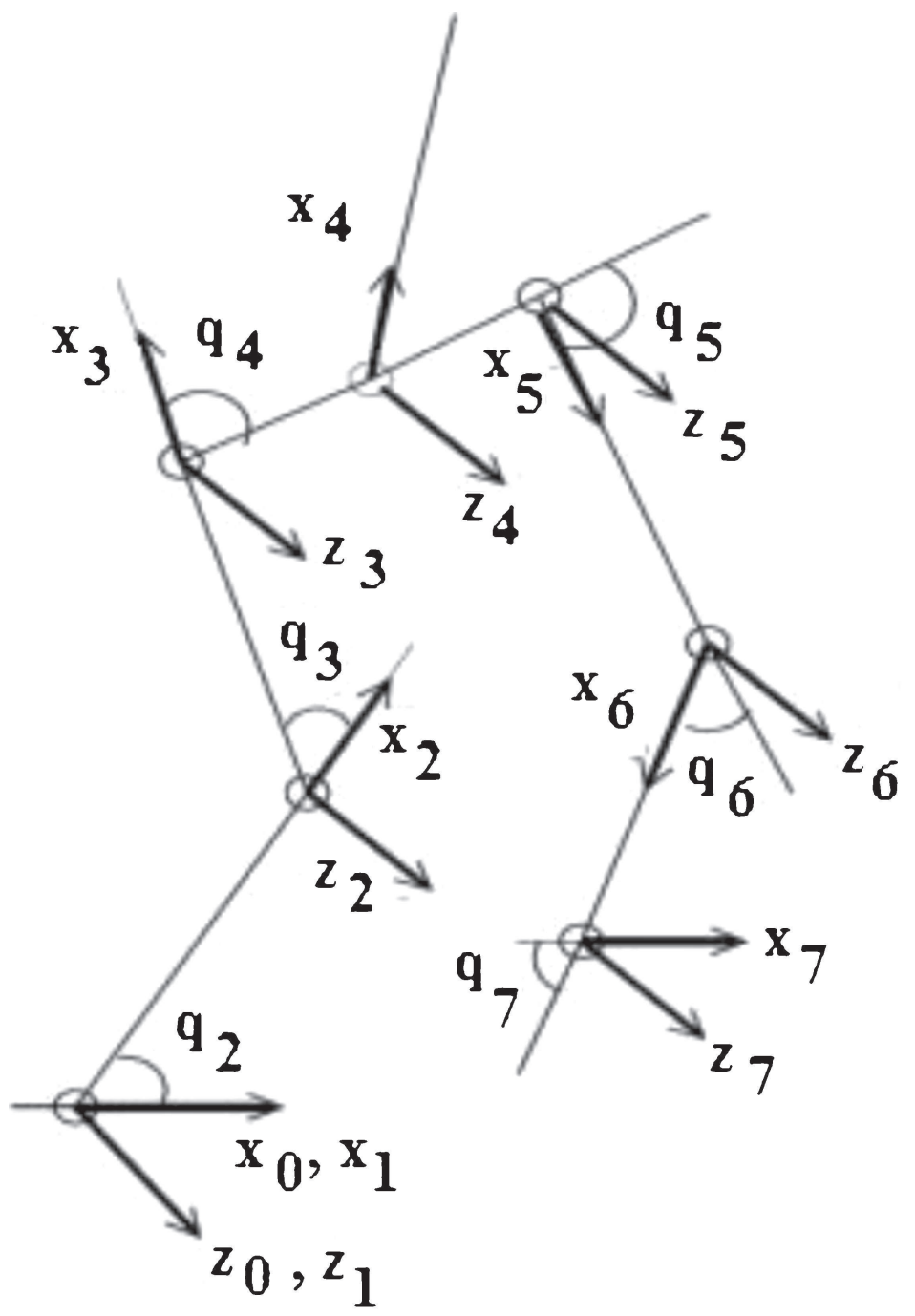

Figure 3. A schematic view showing D-H parameters setting.

respectively. The external force vector $F$ acting at the centre of trunk mass is assumed to have the components as $\left[F_{x}, F_{y}, F_{z}\right]$. There are six equilibrium equations that balance the forces and moments, as given below.

$$
\begin{gathered}
\sum_{i=1}^{7} m_{i} \ddot{x}_{i}+F_{X}+f_{X}=0 \\
\sum_{i=1}^{7} m_{i} \ddot{y}_{i}+F_{y}+f_{y}=0 \\
\sum_{i=1}^{7} m_{i}\left(\ddot{z}_{i}-g\right)+F_{z}+f_{z}=0
\end{gathered}
$$




$$
\begin{aligned}
& -\sum_{i=1}^{7} m_{i} \ddot{y}_{i} z_{i}+\sum_{i=1}^{7} m_{i}\left(\ddot{z}_{i}-g\right) y_{i}+\sum_{i=1}^{7} I_{i} \alpha_{i}+F_{z} y_{4}-F_{y} z_{4}+f_{z} Y_{Z M P}-f_{y} Z_{Z M P}=0 \\
& -\sum_{i=1}^{7} m_{i}\left(\ddot{z}_{i}-g\right) x_{i}+\sum_{i=1}^{7} m_{i} \ddot{x}_{i} z_{i}+\sum_{i=1}^{7} I_{i} \alpha_{i}+F_{x} z_{4}-F_{z} x_{4}+f_{x} Z_{Z M P}-f_{z} X_{Z M P}=0 \\
& -\sum_{i=1}^{7} m_{i} \ddot{x}_{i} y_{i}+\sum_{i=1}^{7} m_{i} \ddot{y}_{i} x_{i}+\sum_{i=1}^{7} I_{i} \alpha_{i}+F_{y} x_{4}-F_{x} y_{4}+f_{y} X_{Z M P}-f_{x} Y_{Z M P}=0
\end{aligned}
$$

The Eqs. (11-13) represent force equilibrium equations and Eqs. (14-16) denote moment equilibrium equations, which are written with respect to the ankle joint. It is to be mentioned that as the motion of the robot is considered in the sagittal plane only, Eq. (12) will have no contribution in the analysis. The stability of the biped robot had been determined using a composition method, where the robot was standing on flat surface Li et al (1991). It is important to mention that ground reaction force acts through the ZMP. The ground reaction force vectors, namely $f_{1}$, $f_{2}$ acting on the ZMPs of the left and right feet were combined to form a DSP and consequently, the system's ZMP ( $\left.X_{Z M P, \text { system }}\right)$ (refer to Eq. (17)) is determined by considering the intersection point of the two forces. A different situation arises in the present study, where the biped robot in DSP stands on two parallel (non-coincidental) surfaces, as shown in figure 1. Sardain \& Bessonnet (2004) propounded the existence of a virtual ZMP for the biped robot's stability in DSP on non-coincidental planes. They extended the concept of Li et al (1991) for the biped robot's DSP to two non-coincidental and coincidental surfaces. In the present study, the DSP of the biped robot standing on two feet placed on two parallel planes has been assumed to be consisting of two SSPs. At each SSP (refer to figure 2), the ZMP is determined using Eq. (9). Using the ground reaction force acting under each foot, the ZMP of the whole robotic system (that is, $X_{Z M P \text {,system }}$ ) is derived, as shown in figure 2 . The expression for $X_{Z M P \text {, system }}$ has been obtained by considering the intersection of the directions of two forces $f_{1}$ and $f_{2}$ (that is, $Z$-heights of the directions of two force vectors are equal at the point of intersection) as given below.

$$
X_{Z M P, \text { system }}=\frac{\hat{f}_{2}-\hat{f}_{1}+\tan \left(\theta_{l}\right) X_{Z M P_{1}}-\tan \left(\theta_{r}\right) X_{Z M P_{2}}}{\tan \left(\theta_{l}\right)-\tan \left(\theta_{r}\right)} .
$$

Here, $\hat{f}_{1}$ and $\hat{f}_{2}$ represent the directions (that is, straight lines on $X-Z$ plane) of the forces: $f_{1}$ and $f_{2}$ (which could be resolved into $X$ and $Z$ directions only). It is to be noted that in Eq. (17), the dimensions of $\hat{f}_{1}$ and $\hat{f}_{2}$ are nothing but that of position. Moreover, the ground reaction forces $f_{1}$ and $f_{2}$ make the angles $\theta_{l}=\tan ^{-1}\left(\hat{f}_{z_{1}} / \hat{f}_{x_{1}}\right)$ and $\theta_{r}=\tan ^{-1}\left(\hat{f}_{z_{2}} / \hat{f}_{x_{2}}\right)$, respectively, with the $X$-direction. The external forces $\left(F_{y}, F_{z}\right)$ on the trunk mass are assumed to be equal to zero. In a very special situation of DSP, when two ground reaction forces become parallel to one another, say in case of static standing, there is no finite intersection point. Here, it is assumed that the system ZMP coincides with the vertically projected point of the centre of mass (COM) 
of the whole body. Dynamic balance margin (DBM) of the robotic system in its DSP can be determined as follows:

$$
D B M_{\text {system }}=\frac{s_{w}-x_{1}+x_{2}+f_{s}}{2}-\left|X_{Z M P, \text { system }}\right| .
$$

\subsection{Staircase descending}

Figure 4 displays the schematic view of a biped robot descending a staircase in double support phase. It is to be noted that fourth link of figure 4 is massless, which, in fact, represents the hip. Three joints are assumed to be coinciding at the hip. The swing foot of the biped robot at the end of its SSP lands on the surface without impact and exerts a force on the ground, thus, enters the DSP. The trunk mass $\left(m_{4}\right)$ is distributed on two supporting legs (left and right). The lengths of projection of trunk mass $m_{4}$ in the double support region are denoted by $X_{1}$ and $X_{2}$ (refer to figure 1). For simplicity, the movement of the biped robot and its balance is considered in one direction only. The hip trajectory has been assumed in the similar way, as it has been done for the ascending case. It is important to note that a similar expression given in Eq. (9) can be used for determination of ZMP, but $g$ has to be replaced by $-g$ in this expression. The generalized angles are to be determined as given below.

$$
\begin{gathered}
q_{1}=\theta_{1} ; q_{2}=\left(-90+\theta_{2}\right) ; q_{3}=\left(\theta_{3}-\theta_{2}\right) ; q_{4}=\left(\theta_{4}-\theta_{3}\right) ; \\
q_{5}=\left(\theta_{5}-\theta_{4}\right) ; q_{6}=\left(\theta_{6}-\theta_{5}\right) ; q_{7}=\left(\theta_{7}-\left(90+\theta_{6}\right)\right) .
\end{gathered}
$$

The aim of this study is to minimize power consumption, after keeping a maximum value of dynamic balance margin. As these two objectives contradict one another, Pareto-optimal front of solutions may exist. Thus, this problem may be treated as a multi-objective optimization problem. Both unconstrained and constrained optimization problems have been solved as discussed below.

\section{Case 1. Unconstrained gait planning for ascending and descending the staircase}

This problem may be mathematically stated as follows:

Minimize power consumption

$$
P_{i}=\frac{1}{T} \sum_{i=1}^{n} \int_{0}^{T}\left(\left|\tau_{i} \dot{q}_{i}\right|+K \tau_{i}^{2}\right) d t
$$

and Minimize

$$
1 / D B M_{\text {system }}=1 /\left(\frac{s_{w}-x_{1}+x_{2}+f_{s}}{2}-\left|X_{Z M P, \text { system }}\right|\right)
$$

subject to

$$
\begin{gathered}
r_{i}^{\min } \leq r_{i} \leq r_{i}^{\max }, \quad \text { where } \quad i=1,2,3,4 \\
m_{4}^{\min } \leq m_{4} \leq m_{4}^{\max }
\end{gathered}
$$




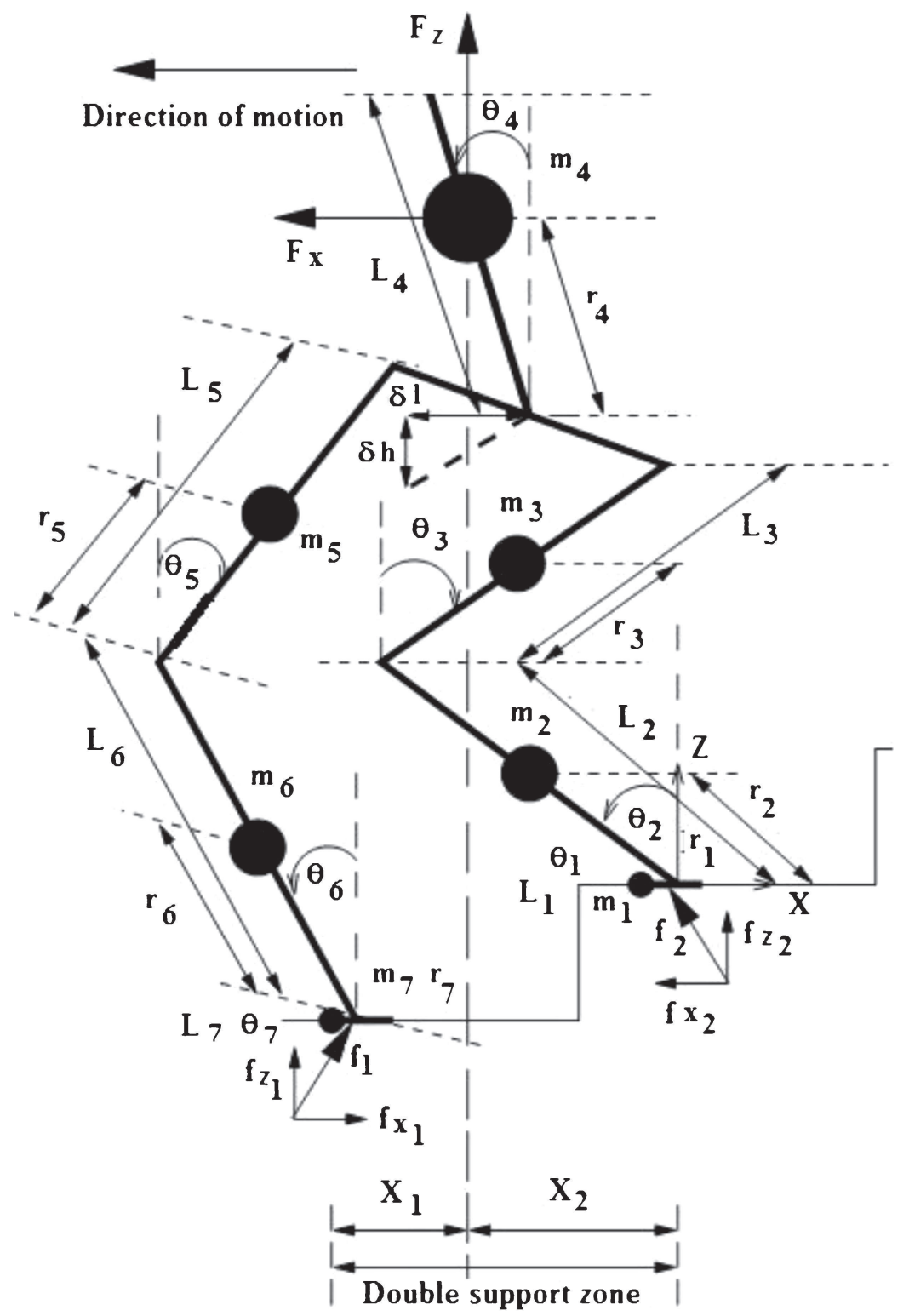

Figure 4. A schematic view showing descending of a 7-dof biped robot.

The parameters: $r_{1}, r_{2}, r_{3}$ and $r_{4}$ denote the mass center positions of first, second, third and fourth links, respectively, and $m_{4}$ represents the trunk mass, $T$ indicates cycle time and $n$ represents the number of joints. It is important to mention that the parameters like $r_{i}(i=1,2,3,4)$ and $m_{4}$ have been considered to be the design variables during optimization. Due to symmetry of the biped robot, $r_{5}, r_{6}$ and $r_{7}$ have been kept equal to $r_{3}, r_{2}$ and $r_{1}$, respectively (refer to figures 1 and 4). 


\section{Case 2. Constrained gait planning for ascending and descending staircase}

In order to avoid jerky motion of the robot, the change in joint torque at each step of a cycle should be less than some pre-specified small value. It has been considered as a functional constraint of this optimization problem. This problem may be stated as follows:

Minimize power consumption

$$
P_{i}=\frac{1}{T} \sum_{i=1}^{n} \int_{0}^{T}\left(\left|\tau_{i} \dot{q}_{i}\right|+K \tau_{i}^{2}\right) d t
$$

and Minimize

$$
1 / D B M_{\text {system }}=1 /\left(\frac{s_{w}-x_{1}+x_{2}+f_{s}}{2}-\left|X_{Z M P, \text { system }}\right|\right)
$$

subject to

$$
\Delta \tau_{i j} \leq \Delta \tau_{\text {specified }}
$$

and

$$
\begin{gathered}
r_{i}^{\min } \leq r_{i} \leq r_{i}^{\max }, \quad \text { where } \quad i=1,2,3,4 \\
m_{4}^{\min } \leq m_{4} \leq m_{4}^{\max }
\end{gathered}
$$

Here, $\Delta \tau_{i j}$ represents the change in torque of $i^{t h}$ joint at $j^{t h}$ time interval. It is to be noted that a violation of this functional constraint changes the torque requirement of the joint suddenly. Due to this sudden change in torque requirement, the motor connected to this joint may be overloaded and consequently, it may have jerky motion and fail. It is also to be noted that the parameters, namely $r_{i}(i=1,2,3,4)$ and $m_{4}$ are considered as the design variables during optimization.

\section{Proposed algorithm}

The DSP starts at the end of SSP in a walking cycle. The problems of gait planning in DSP of a biped robot ascending and descending the staircase have been studied as multi-objective optimization ones. For the purpose of analysis, the trunk mass $\left(m_{4}\right)$ is distributed into $m_{41}$ and $m_{42}$ depending on its position in the DSP (refer to figures 1 and 4). The ZMP is calculated for each of the ground feet, such as $X_{Z M P_{1}}$ for left leg and $X_{Z M P_{2}}$ for right leg, respectively, to form a DSP of the biped robot for staircase gait planning. In the DSP of the robot, two conflicting objectives, namely power consumption $(P)$ and $D B M_{\text {system }}$ have been determined.

The robot should be able to maintain more dynamic balance margin at the cost of less power consumption. However, in order to have more balance margin, more power is to be consumed to ensure sufficient amount of rotation of the joint links to reach the desired configuration of the robot. Therefore, the above two objectives are conflicting one another. The problem of multiobjective optimization has been solved using a genetic algorithm named NSGA-II Deb et al (2002) and a particle swarm optimization algorithm called MOPSO_CD (Raquel \& Naval 2005; www.particleswarm.info). Both the algorithms start with a population of initial solutions generated at random. It is to be noted that the terminal positions of the joints in SSP have been 
considered as the starting values of joint angles in DSP. The biped robot's DSP motion on noncoincidental planes is achieved by a small hip movement of $\delta l$ and $\delta h$ in $X$ and $Z$ directions, respectively (refer to figure 1).

\subsection{Genetic algorithm}

Genetic Algorithm (GA) (Holland 1975; Goldberg 1989) is a population-based probabilistic search technique, which works based on the principle of natural genetics. The genetics itself relies on the survival of the fittest. The evolution occurs through reproduction, crossover, and mutation. The GA has been used for solving multi-objective optimization problem also. Nondominating Sorting Genetic Algorithm (NSGA-II) is one of such algorithms.

Initially, a random population of solutions is generated. Each solution is compared with the others lying in the population in terms of its fitness to find, if it is dominated. In this regard, two entities are calculated, namely (i) domination count $n_{p}$, that is, the number of solutions, which dominate the solution $\mathrm{p}$, and (ii) $s_{p}$, a set of solutions that the solution $\mathrm{p}$ dominates. Now, using the concept of $n_{p}$ and $s_{p}$, the whole population of solutions is divided into some fronts like first, second, third, and so on.

In order to preserve good spread in the obtained set of solutions, a concept of crowding distance is utilized. A crowding comparison is used to test as density estimation metric, that is, to get an estimation of the density of solutions surrounding a particular solution in the population. The average distance of two points on either side of this point along each of the objectives is calculated. This distance is nothing but the perimeter of the cuboid formed using the nearest neighbours as the vertices, which is known as crowding distance. The crowding distance values are used for sorting the population according to each objective function value in ascending order of magnitude. Therefore, for each objective function, the boundary solutions (solutions with the smallest and largest function values) are assigned an infinite distance value. All other intermediate solutions are assigned distance values equal to the absolute normalized differences in the function values of two adjacent solutions. This calculation is continued with other objective functions. The overall crowding distance value is calculated as the sum of the individual distance value corresponding to each objective. Each objective function is normalized before determining the crowding distance. For all solutions in the non-dominated set, initialize the distance for each solution to zero. For each objective function (fitness) value, sort all other non-dominated values in descending order. The crowded-comparison selection process at the various stages of the algorithm guides towards uniformly spread-out Pareto-optimal front using non-domination rank and crowding distance. For two solutions with different non-domination ranks, the one with the lower rank will be selected. Otherwise, if both solutions belong to the same front (that is, rank) the solution located in a less crowded region is selected.

This process is repeated until the number of generation reaches the pre-specified maximum number of generations. NSGA-II algorithm (Deb et al 2002) is also capable of handling constrained optimization problems. A concept of fixed penalty function approach (Pratihar 2008) has been used, if there is a violation of any constraint.

The following steps are used in NSGA-II algorithm:

1. A random population of solutions is generated.

2. Solutions are sorted for non-domination and then ranked as rank 1 , rank $2, \ldots$, etc.

3. Reproduction is implemented.

4. Crossover is used to bring changes in the solutions.

5. Mutation is implemented to bring a local change in the solutions. 
6. To maintain the diversity and spread in the ranks, the concept of crowding distance is utilized. Every individual solution is ranked. If the solutions belong to different ranks, then the solution with the lower (that is, the better) rank is preferred. If the comparing solutions belong to the same rank, the solution located in the less crowded region is preferred.

The GA will try to determine the solution corresponding to the maximum dynamic balance margin of the robot but at the expense of minimum power. The power consumption and dynamic balance margin of the robot have been calculated to formulate it as a multi-objective optimization problem, and accordingly, the fitness values of GA-solutions have been calculated.

\subsection{Particle swarm optimization algorithm}

Particle Swarm Optimization (PSO) is a technique, which works based on the concept of swarm behaviour in searching food in the neighbourhood space efficiently and effectively. The particle is defined with respect to its two parameters, namely position and velocity in search space. The $i^{\text {th }}$ particle's position and velocity vectors in $d$-dimensional search space can be represented as $X_{i}=\left(x_{i 1}, \ldots \ldots ., x_{i d}\right)$ and $V_{i}=\left(v_{i 1}, \ldots \ldots \ldots, v_{i d}\right)$, respectively. The value of $V_{i}$ vector can be varied in the range of $\left[-v_{\max }, v_{\max }\right]$ to reduce the tendency of particles to leave the search space. The value of $v_{\max }$ is usually chosen to be equal to $k \times x_{\max }$, where $0.1 \leq k \leq 1.0$ (Eberhert et al 1996). The trajectory of each individual in the search space is adjusted dynamically according to its own flying experience and information provided by other particles in the search space. The swarm's global best solution is achieved simply by adjusting the trajectory of each individual toward its own best location and the best particle of the entire swarm at each time step (also known as generation) (Kennedy \& Eberhart 1995; Clerc \& Kennedy 2002). For a given fitness function, each particle's best solution (Pbest) at time $t$ is given as $P_{i}=\left(p_{i 1}, \ldots \ldots ., p_{i d}\right)$ and the swarm's fittest particle (Gbest) during the same time $t$ is denoted by $P_{g}=\left(p_{g 1}, \ldots \ldots, p_{g d}\right)$. The new velocities and positions of the particles for the next fitness evaluation are calculated using the following two equations:

$$
\begin{gathered}
v_{i d}(t+1)=W v_{i d}(t)+c_{1} \text { rand }(\cdot)\left(p_{i d}-x_{i d}(t)\right)+c_{2} \text { Rand }(\cdot)\left(p_{g d}-x_{i d}(t)\right), \\
x_{i d}(t+1)=x_{i d}(t)+v_{i d}(t+1),
\end{gathered}
$$

where $v_{i d}$ is the velocity of $d^{\text {th }}$ dimension of $i^{\text {th }}$ particle, $W$ is a constant known as inertia weight (Shi \& Eberhart 1999), $c_{1}$ and $c_{2}$ denote the acceleration coefficients, and rand (.) and Rand $(\cdot)$ are two separately generated uniformly distributed random numbers lying in the range of $\{0,1\}$. The first part of Eq. (19) represents the previous velocity that provides the necessary momentum to the particles to move across the search space. The second part of the Eq. (19) represents cognitive component responsible for personal thinking of each particle. It helps the particles to move toward their respective best solutions. The third part of Eq. (19) indicates social component, which controls collaborative effect of the particles in order to find the globally best solution. The PSO is able to gain much attention nowadays due to its simple architecture, ease of implementation and ability to quickly reach the global optimal solution.

Multi-objective PSO (that is, MOPSO-CD) (Raquel and Naval 2005; www.particleswarm. info) incorporates the mechanism of crowding distance of NSGA-II (Deb et al 2002) into the PSO. The concept of crowding distance together with mutation operator maintains the diversity of non-dominated solutions. The MOPSO-CD can also handle constrained optimization 
problems. The working principle of MOPSO-CD can be stated in steps as follows (Das \& Dehuri 2011):

(i) A population of particles is generated at random, whose velocities are set equal to zero.

(ii) Each particle's best fitness (Pbest), swarm's best fitness (Gbest) are computed and stored in archive. The crowding distance values are calculated and sorted in descending order. A solution is randomly selected as Gbest from the top (10\%) of the values. Gbest acts as the global best guide for the non-dominated solutions.

(iii) Velocities and positions of the population of particles are updated.

(iv) Non-dominated solutions obtained from the updates are inserted into the archive. All dominated solutions are deleted from the archive. The crowding distance values are computed and sorted in descending order. Select randomly the most crowded values (bottom 10\%) as the particle's Pbest. Update the particle's Pbest.

(v) Repeat iterations.

As discussed above, the values of two objectives, namely power consumption and dynamic balance margin are dependent on ten variables, which have been coded in the PSO-solutions. The fitness values of PSO-solutions are determined corresponding to the said two objectives, as these are calculated for the GA-solutions. A penalty function approach has been adopted in order to penalize a solution, if there is a violation of functional constraint.

\section{Results and discussion}

The biped robot consists of links with the masses (in $\mathrm{kg}$ ): $m_{1}=m_{7}=0.5 ; m_{2}=m_{6}=2.0$; $m_{3}=m_{5}=5.0$ and $m_{4}$ is varied in the range from 10.0 to $50.0 \mathrm{~kg}$. The links are assumed to have the lengths (in $\mathrm{m}$ ) as follows: $l_{1}=l_{7}=0.06, l_{2}=l_{6}=0.34, l_{3}=l_{5}=0.30, l_{4}=0.6$. A cycle time of three seconds has been divided into three equal time intervals. During optimization, the ranges of design variables: $r_{1}, r_{2}, r_{3}, r_{4}$ and $m_{4}$ are kept equal to $(0.01,0.02),,(0.1,0.32)$, $(0.1,0.28),(0.1,0.54)$ and $(10.0,50.0)$, respectively. In simulations, the other parameters, such as $\theta_{2}, \theta_{3}, \theta_{4}, \delta l$ and $\delta h$ are varied in the ranges of $(33.25,36.75),(-84.00,-76.00),(11.74$, $12.98),(0.0027,0.003)$ and $(0.0027,0.0033)$, respectively. Computer simulations are conducted on a P-IV PC.

\subsection{Results of unconstrained optimization}

Results of unconstrained multi-objective optimization problems related to ascending and descending the staircases, as obtained by the GA and PSO algorithm separately, have been stated and discussed below.

4.1a Ascending the staircase: As the performances of both the GA and PSO algorithm are dependent on their respective parameters' values, a thorough parametric study has been carried out for each of them, separately. In this study, only one parameter has been varied at a time, keeping the others fixed. The following GA-parameters are found to give the best results: crossover probability $p_{c}=0.82$; mutation probability $p_{m}=0.0055$; maximum number of generations $=$ 100 and population size $=100$. Similarly, the following PSO parameters have obtained the best results: number of runs $=100$; swarm size $=100$. The GA has obtained the optimized values of the design variables as follows: $r_{1}=0.015 \mathrm{~m}, r_{2}=0.22 \mathrm{~m}, r_{3}=0.18 \mathrm{~m}, r_{4}=0.45 \mathrm{~m}$, 
$m_{4}=35 \mathrm{~kg}$. On the other hand, the optimal values of the design variables are found to be as $r_{1}=0.016 \mathrm{~m}, r_{2}=0.24 \mathrm{~m}, r_{3}=0.18 \mathrm{~m}, r_{4}=0.35 \mathrm{~m}, m_{4}=38 \mathrm{~kg}$ by the PSO algorithm. Figure 5 displays the variations of $X_{Z M P \text {, system }}$ in a cycle as obtained by the GA- and PSO-based optimization separately. It shows that the robot is able to maintain its dynamic balance in the cycle using the optimal parameters yielded by the GA and PSO algorithm, separately. Moreover, the PSO algorithm is able to provide more dynamically balanced gaits of the robot compared to the GA. Figure 6 shows the Pareto-optimal fronts of solutions obtained by the GA and PSO algorithm for the staircase ascending problem. It is interesting to observe that the GA has been defeated by PSO algorithm in terms of the quality of Pareto-optimal front of solutions obtained by them. It may have happened due to the reason that the PSO algorithm can carry out both the global and local searches simultaneously; whereas the GA is weak in its local search capability, although it is a potential tool for global optimization. Moreover, the particles (solutions) in PSO algorithm use their memory and intelligence, while moving from one population to the next, which is missing in the GA-search. It is to be noted that any point lying on the Pareto-optimal front is an optimal solution yielded by considering a particular set of weights on two objective functions. Thus, the designer will have a choice to select the appropriate optimal solution out of all the points lying on the Pareto-optimal front.

4.1b Descending the staircase: The optimized GA- and PSO-parameters have been obtained separately for the problems of staircase descending also. The best results are yielded with the following GA-parameters: crossover probability $p_{c}=0.88$, mutation probability $p_{m}=0.0060$, maximum number of generations $=100$ and population size $=100$. The GA has yielded the optimal values of the design variables as $r_{1}=0.011 \mathrm{~m}, r_{2}=0.21 \mathrm{~m}, r_{3}=0.25 \mathrm{~m}, r_{4}=0.37 \mathrm{~m}$,

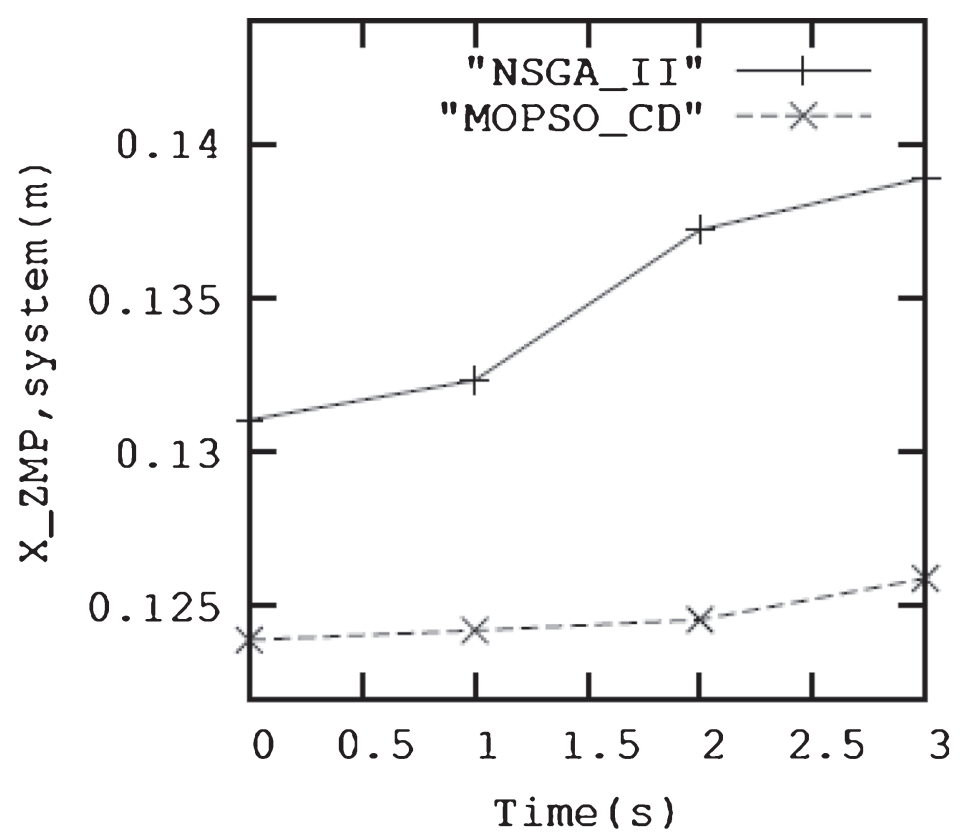

Figure 5. Variations of $X_{Z M P, \text { system }}$, as obtained by the GA and PSO algorithm separately for unconstrained optimization in staircase ascending problems. 


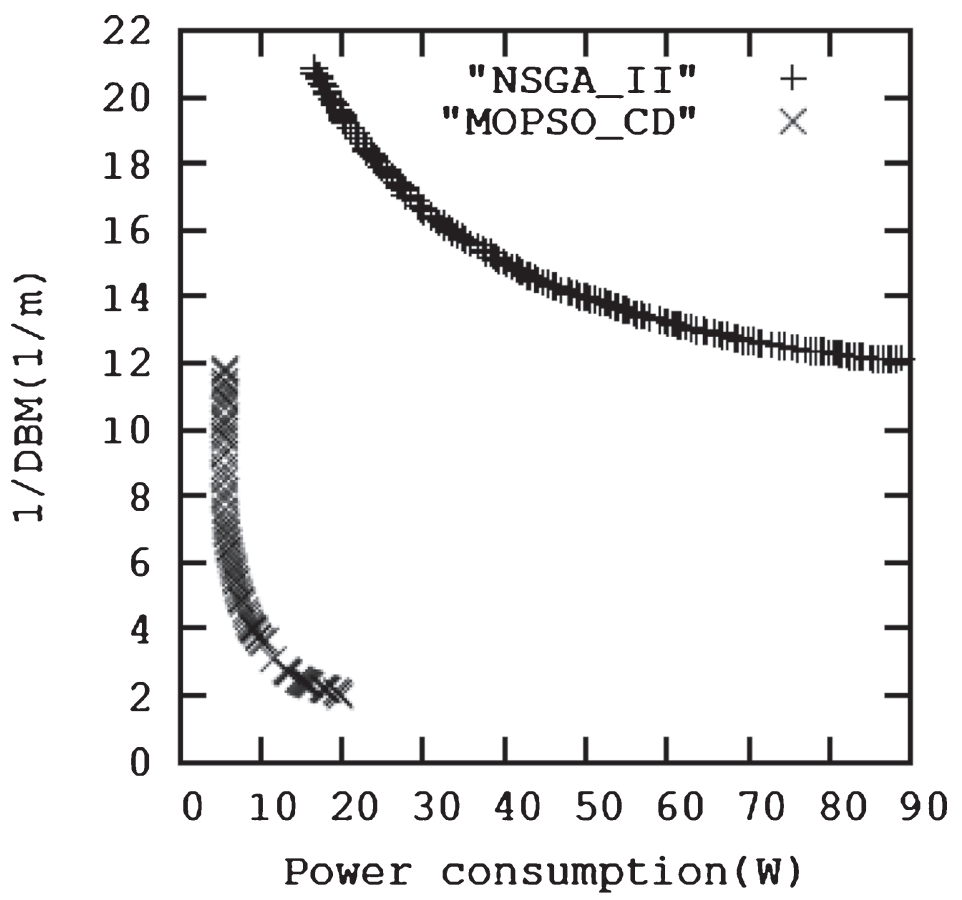

Figure 6. Pareto-optimal fronts of solutions obtained by the GA and PSO algorithm separately for unconstrained optimization in staircase ascending problems.

$m_{4}=39 \mathrm{~kg}$. Moreover, the best results have been obtained for the following PSO-parameters: number of runs $=100$, swarm size $=100$. The optimized values of the design variables are seen to be as follows: $r_{1}=0.015 \mathrm{~m}, r_{2}=0.26 \mathrm{~m}, r_{3}=0.18 \mathrm{~m}, r_{4}=0.38 \mathrm{~m}, m_{4}=46 \mathrm{~kg}$. Figure 7 shows the variations of $X_{Z M P \text {, system. The Pareto-optimal fronts of solutions obtained }}$ by the GA and PSO algorithm separately, for this problem, are shown in figure 8. Once again, the PSO algorithm has outperformed the GA in terms of the quality of Pareto-optimal front of solutions. It may be so, due to the reasons mentioned above. The minimum power consumption of the robot, is found to be equal to $7.02128 \mathrm{~W}$ (figure 8).

4.1c Discussion: The left top-most point of the Pareto-optimal front of solutions obtained by the GA and PSO separately, as shown in figures 6 and 8 correspond to the minimum power consumption and DBM, whereas the maximum values of power consumption and DBM are indicated by the right-most point of the said figures. Moreover, the trunk mass is found to reach its minimum and maximum values at the left- and right-most points of the Pareto-optimal front of solutions, respectively. Thus, both power consumption as well as DBM is seen to increase with the trunk mass. It is in line with the experiences of human-beings ascending and descending the staircases.

Figures 6 and 8 indicate that for a particular value of power consumption, DBM is more in descending gaits compared to that of ascending gaits. Moreover, ascending gaits have been found to consume more power in comparison with the descending gaits for a particular value of DBM. These observations match with the general experiences of human-beings. 


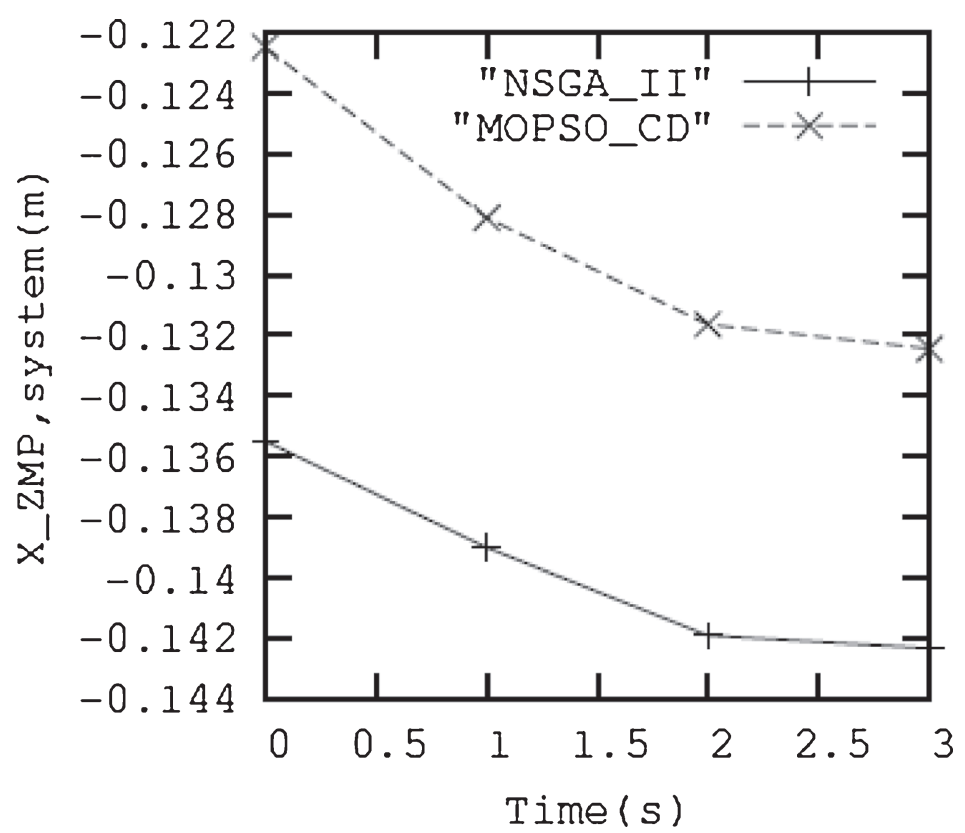

Figure 7. Variations of $X_{Z M P, \text { system }}$ in a cycle (seconds) as obtained by the GA and PSO algorithm for unconstrained optimization in staircase descending problems.

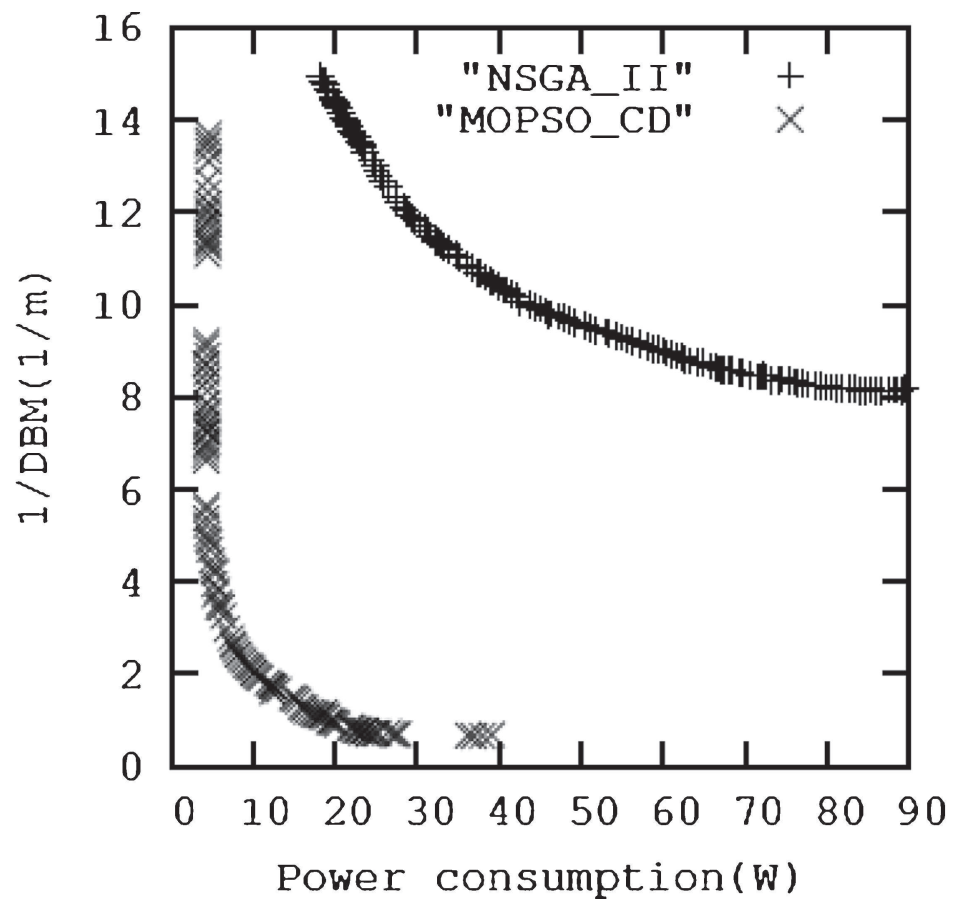

Figure 8. Pareto-optimal fronts of solutions obtained by the GA and PSO for unconstrained optimization in staircase descending problems. 
The performances of the GA and PSO algorithm have been compared through figures 6 and 8 in terms of the quality of Pareto-optimal fronts of solutions obtained by them. The PSO algorithm has shown better performance compared to the GA for the problems of both ascending and descending gait generations. It has happened so, due to the reasons discussed above. Moreover, the PSO is a greedier algorithm compared to the GA.

\subsection{Results of constrained optimization}

Constrained optimization problems related to ascending and descending the staircases have also been tackled using the GA and PSO algorithm, separately. A penalty function approach has been adopted to penalize a solution, if there is a violation of functional constraint. The results are stated, discussed and compared below.

4.2a Ascending the staircase: The following GA-parameters have yielded the best results: $p_{c}=0.78, p_{m}=0.0050$, maximum number of generations $=100$ and population size $=100$. The GA has yielded the optimized values of the design variables as $r_{1}=0.011 \mathrm{~m}, r_{2}=0.21 \mathrm{~m}$, $r_{3}=0.22 \mathrm{~m}, r_{4}=0.44 \mathrm{~m}, m_{4}=40 \mathrm{~kg}$. Similarly, the following PSO-parameters obtained through a careful study are seen to give the best results: number of runs $=100$, swarm size $=100$. The optimal values of the design variables are found to be as follows: $r_{1}=0.015 \mathrm{~m}, r_{2}=0.31 \mathrm{~m}$, $r_{3}=0.23 \mathrm{~m}, r_{4}=0.34 \mathrm{~m}, m_{4}=44 \mathrm{~kg}$. The variations of $X_{Z M P, \text { system }}$ in a cycle are shown in figure 9. The Pareto-optimal fronts of solutions as obtained by the GA and PSO algorithm separately for this problem, (figure 10). The PSO algorithm has outperformed the GA again, and the reasons behind this fact have been explained above. Any point present on this front is an optimal

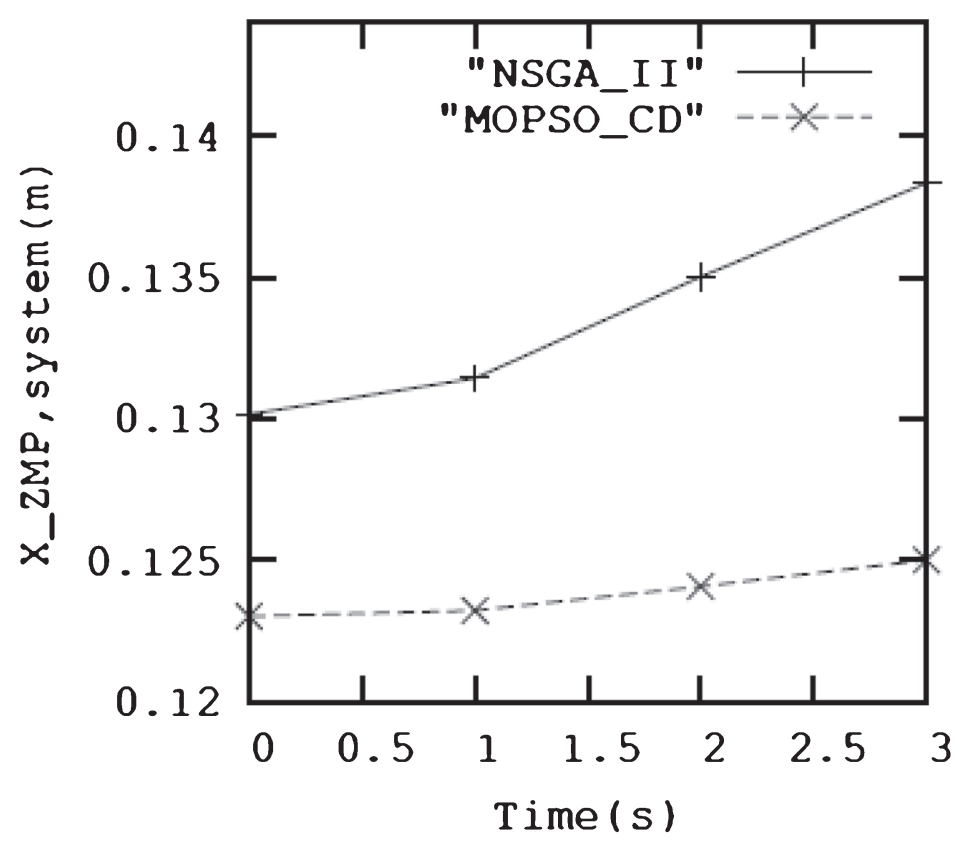

Figure 9. Variations of $X_{Z M P, \text { system }}$ in a cycle as obtained by the GA and PSO algorithm for constrained optimization in staircase ascending problems. 


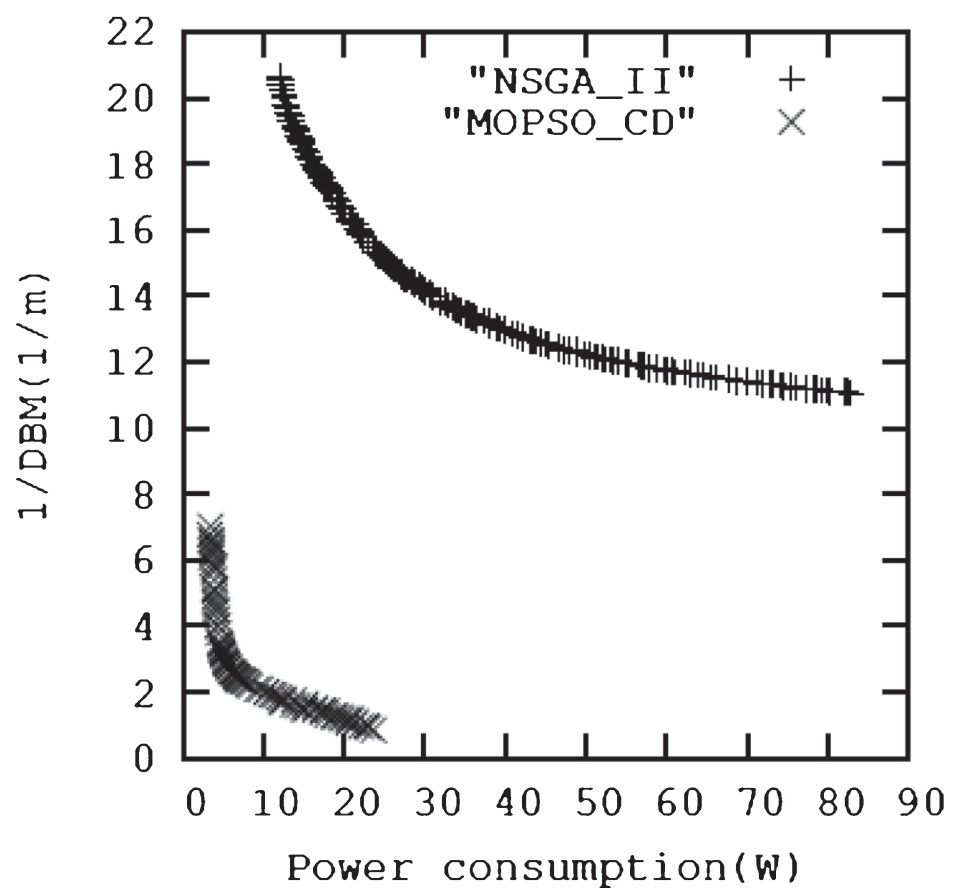

Figure 10. Pareto-optimal fronts of solutions obtained by the GA and PSO algorithm for constrained optimization in staircase ascending problems.

solution obtained after assigning a particular set of weights on two objective functions. Thus, the designer will have a choice to select an appropriate optimal solution out of all the points lying on the Pareto-optimal front.

Figure 11 shows the movements of different links and joints of the biped robot during its double support phase, while ascending the staircases. The duration for double support phase has been assumed to be equal to 3.0 seconds, and this figure shows the positions of different links of the biped robot at the end of first, second and third seconds.

4.2b Descending the staircase: The optimal set of GA-parameters has been obtained through a parametric study as follows: crossover probability $p_{c}=0.86, p_{m}=0.005$, maximum number of generations $=100$ and population size $=100$. The GA has determined the optimal values of the design variables as follows: $r_{1}=0.018 \mathrm{~m}, r_{2}=0.28 \mathrm{~m}, r_{3}=0.22 \mathrm{~m}, r_{4}=0.34 \mathrm{~m}, m_{4}=41 \mathrm{~kg}$. The optimal PSO-parameters are found to be like the following: number of runs $=100$, swarm size $=100$. The PSO algorithm has obtained the optimized values of the variables as $r_{1}=0.012 \mathrm{~m}$, $r_{2}=0.22 \mathrm{~m}, r_{3}=0.25 \mathrm{~m}, r_{4}=0.36 \mathrm{~m}, m_{4}=47 \mathrm{~kg}$. Figures 12 and 13 display the variations of $X_{Z M P, \text { system }}$ in a cycle and Pareto-optimal fronts of solutions, as yielded by the GA and PSO algorithms, respectively. It is to be noted that the PSO algorithm has achieved an optimal solution corresponding to a minimum power consumption of $4.80243 \mathrm{~W}$. Once again, the GA has been defeated by the PSO algorithm in terms of the quality of obtained Pareto-optimal fronts of solutions. 


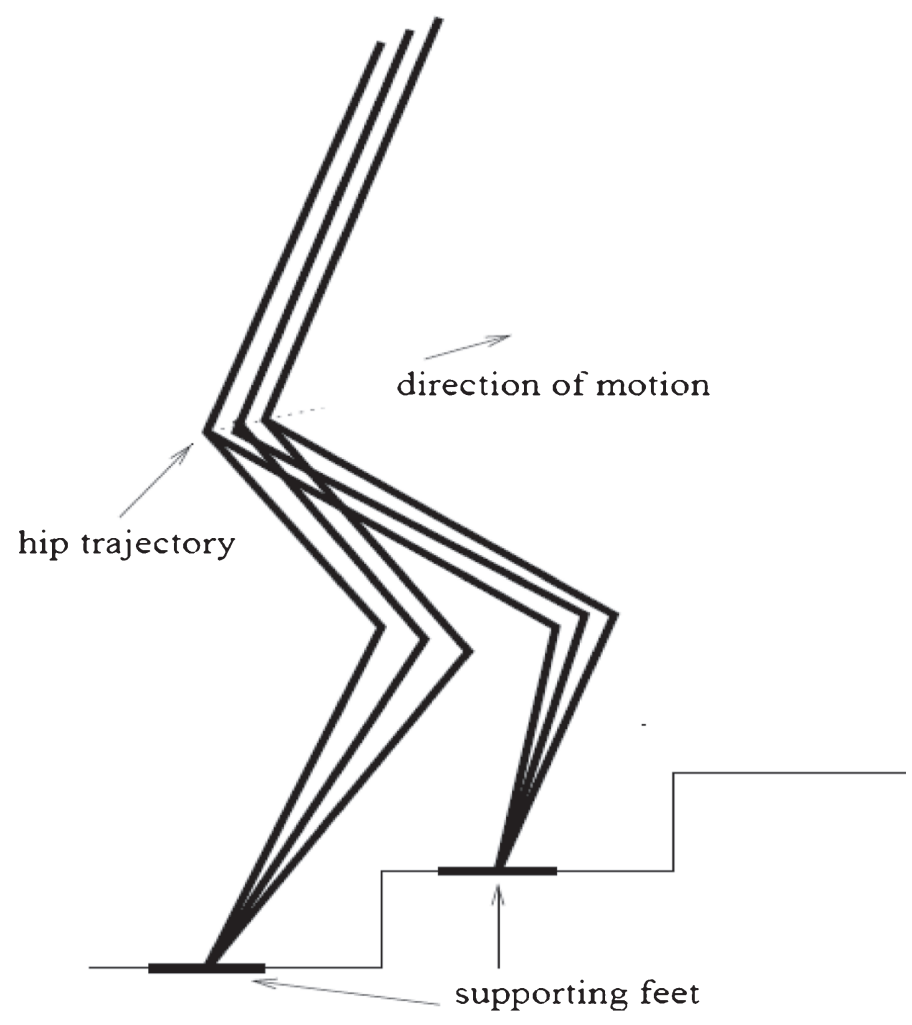

Figure 11. A schematic view showing the movements of different links and joints during its double support phase, while ascending the staircases.

Figure 14 shows the positions of different links at the end of first, second and third seconds during its double support phase, while descending the staircases.

\subsection{Discussion}

A comparison between Figures 10 and 13 indicates that the DBM in descending gait becomes more than that in ascending gait generation for a particular value of power consumption. Moreover, ascending gait requires more power than descending gait does for a particular value of DBM. Once again, the PSO is found to outperform the GA in terms of the quality of obtained Pareto-optimal fronts of solutions. It has happened so, for the reasons given above.

This paper concentrates on the analysis of DSP of walking of a biped robot negotiating staircases. The DSP has been assumed to be consisting of two SSPs for the purpose of analysis. It has been formulated as a multi-objective optimization problem in order to maximize dynamic balance margin of the biped robot after consuming the minimum power, and it has been solved using a GA and PSO algorithm, separately. To the best of the authors' knowledge, it is a novel approach for tackling DSP of biped walking as a multi-objective optimization problem, in which Pareto-optimal fronts of solutions have been obtained. 


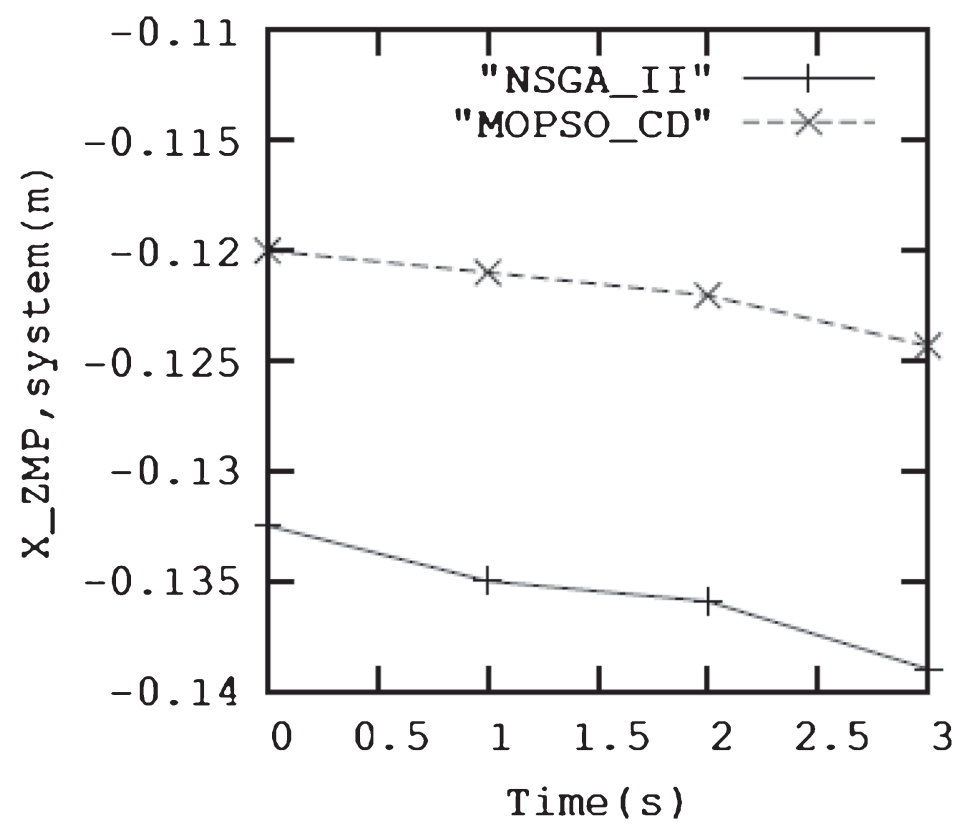

Figure 12. Variations of (a) $X_{Z M P, \text { system }}$ in a cycle as obtained by the GA and PSO algorithm for constrained optimization in staircase descending problems.

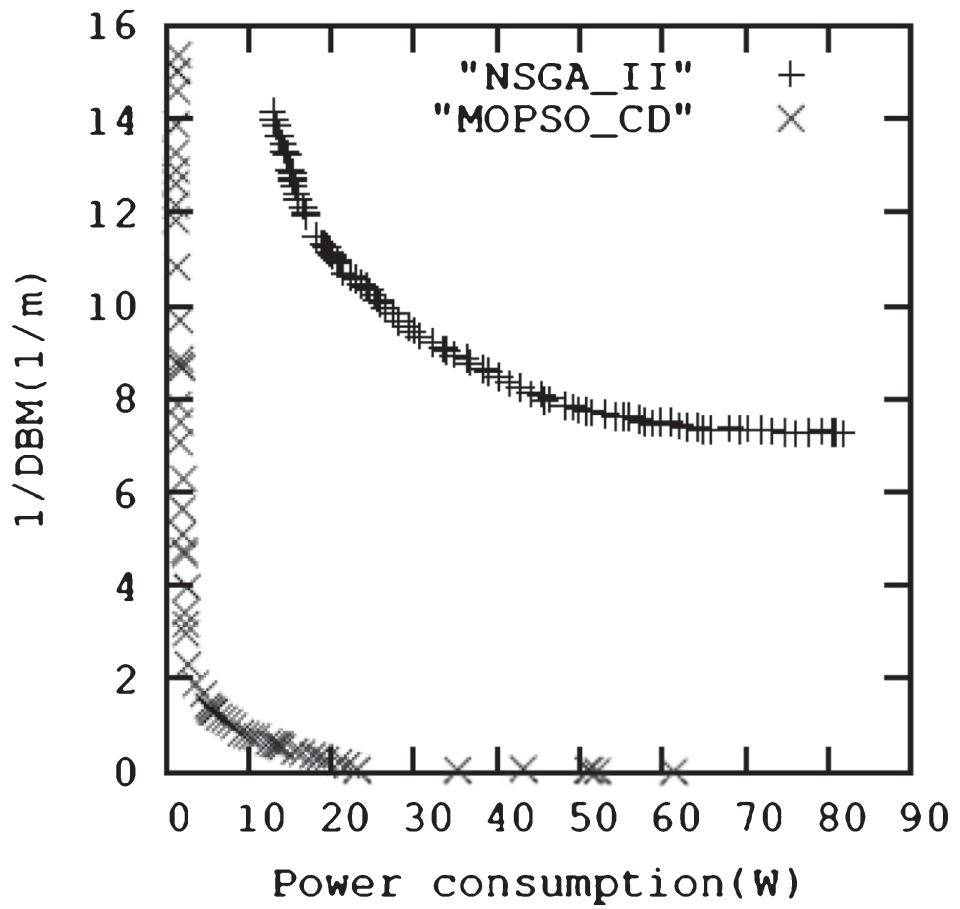

Figure 13. Pareto-optimal fronts of solutions obtained by the GA and PSO algorithm for constrained optimization in staircase descending problems. 


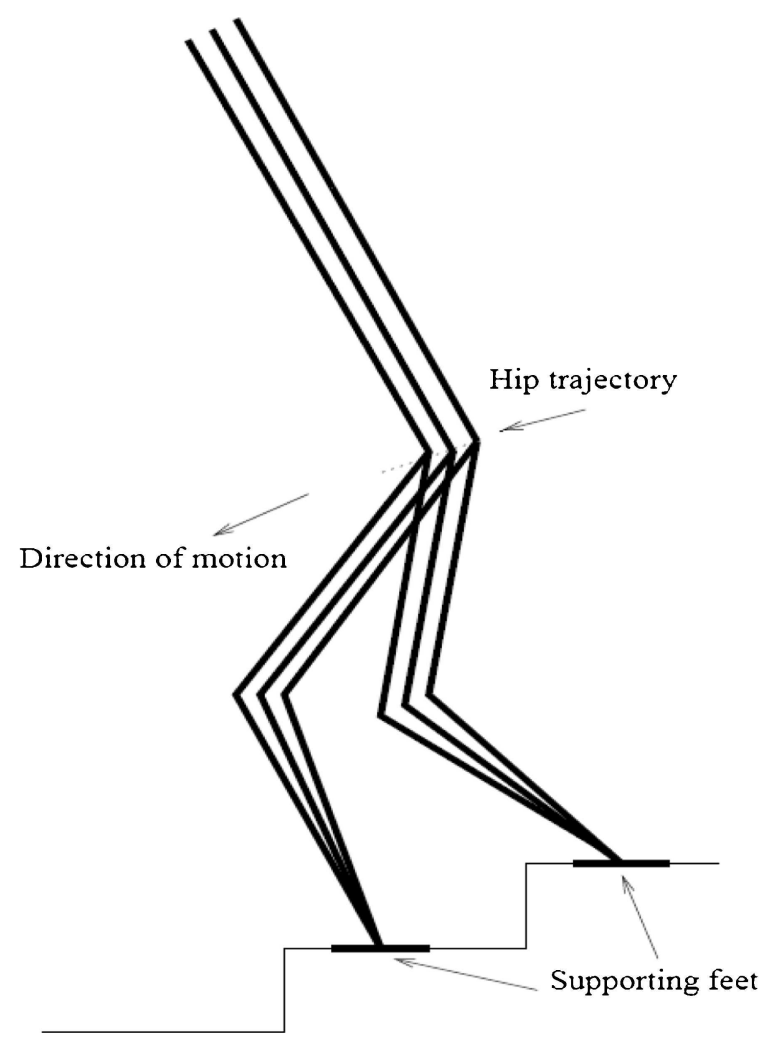

Figure 14. A schematic view showing the movements of different links and joints during its double support phase, while descending the staircases.

\section{Concluding remarks}

This study deals with gait planning problems of a 7-dof biped robot in its double support phase while ascending and descending some staircases after consuming minimum power and maintaining a high dynamic stability margin. As these two objectives contradict each another, it is an ideal problem for multi-objective optimization. Both the constrained as well as unconstrained optimization problems have been solved using the GA and PSO algorithm separately, and Paretooptimal fronts of solutions have been obtained. The findings of this study in terms of power consumption and dynamic stability margin are in tune with the general experience of humanbeings ascending and descending some staircases. The PSO algorithm is found to perform better than the GA. The former carries out both the global and local searches simultaneously, whereas the latter is a potential tool for the global search only. The obtained Pareto-optimal fronts of solutions may help the designer to select some appropriate optimal solutions depending on the requirements. Thus, it helps the designer to arrive at a suitable design of the biped robot. In the present study, a simplified model of biped robot having 7-dof has been considered, whose movement has been restricted in the direction of its travel, that is, $\mathrm{X}$ direction. However, its movement in $\mathrm{Y}$ direction has been neglected for simplicity. A real humanoid robot has its movements in both $\mathrm{X}$ and $\mathrm{Y}$ directions, besides its motion along $\mathrm{Z}$ direction. Therefore, the present model is a simplified version of a real humanoid robot. However, the present analysis may help to carry out 
the similar analysis for a realistic humanoid robot. It has been kept in the scope of future work. Therefore, the proposed method is yet to be validated using a real robot, which will be tried in future. For simplicity, the hip is assumed to follow a straight path (in Cartesian coordinate system) with the slope kept equal to that of the staircase in order to maintain repeatability of the cycle. However, some other trajectories may also be tried for the hip joint and their impacts on power consumption and DBM will be studied in future. In the present study, only one functional constraint, that is, change in joint torques to be within a pre-specified range, has been considered. However, there may be some other functional constraints like acceleration limit and power rating of the motor, and others, which will be studied in future. In the present study, the joint angles are assumed to be cubic polynomials for simplicity. However, fifth-order polynomials might be applied to ensure jerk-free motion of the joints, which has been kept in the scope for future study. The aim of this study is to obtain optimized mechanical structure of the biped robot. However, the problem of biped robot's motion has not been optimized here. It will be tried in future after the real biped robot is manufactured.

\section{Acknowledgements}

The authors thank All India Council of Technical Education (AICTE), Govt. of India, for supporting this study and Mr. Ashutosh Kumar Misra, for providing some useful suggestions.

\section{Appendix A}

\section{Expressions for Joint Torques}

The equations of $\mathrm{n}$ joint torques can be written as follows (Fu et al 1987):

$$
\begin{gathered}
\tau_{i}=\sum_{k=1}^{n} D_{i k} \ddot{q}_{k}+\sum_{k=1}^{n} \sum_{m=1}^{n} h_{i k m} \dot{q}_{k} \dot{q}_{m}+C_{i}, \mathrm{i}=1,2 \ldots, \mathrm{n}, \\
D_{i k}=\sum_{j=\max (i, k)}^{n} \operatorname{Tr}\left(U_{j k} J_{j} U_{j i}^{T}\right), \quad \mathrm{i}, \mathrm{k}=1,2, \ldots, \mathrm{n}, \\
h_{i k m}=\sum_{j=\max (i, k, m)} \operatorname{Tr}\left(U_{j k m} J_{j} U_{j i}^{T}\right), \mathrm{i}, \mathrm{k}, \mathrm{m}=1,2 \ldots, \mathrm{n}, \\
C_{i}=\sum_{j=1}^{n}\left(-m_{j} g U_{j i} \bar{r}_{j}^{j}\right), \mathrm{i}=1,2 \ldots, \mathrm{n},
\end{gathered}
$$

where $D_{i k}$ denotes inertia terms, $h_{i k m}$ represents the Coriolis and centrifugal terms, and $C_{i}$ indicates information of the gravity terms. 


\section{List of symbols}

\begin{tabular}{|c|c|}
\hline$a$ & Acceleration, $\mathrm{m} / \mathrm{s}^{2}$ \\
\hline$a$ & Acceleration, $\mathrm{m} / \mathrm{s}^{2}$ \\
\hline$a_{i 0}^{\prime}, \ldots, a_{i 3}^{\prime}$ & Coefficients of joint angle trajectories \\
\hline$c_{1}, c_{2}$ & Acceleration coefficients \\
\hline$d$ & Search dimension space of a particle \\
\hline$d_{1}, \ldots, d_{7}$ & Distance of mass centres on the limbs, $m$ \\
\hline$f_{s}$ & Length of the foot, $\mathrm{m}$ \\
\hline$f_{1}, f_{2}$ & Ground reaction force, $\mathrm{N}$ \\
\hline$f_{x_{1}}, f_{x_{2}}$ & Friction force, $\mathrm{N}$ \\
\hline$f_{z_{1}}, f_{z_{2}}$ & Normal reaction force, $\mathrm{N}$ \\
\hline$g$ & Acceleration due to gravity, $\mathrm{m} / \mathrm{s}^{2}$ \\
\hline Gbest & Swarm global best solution \\
\hline$h$ & Hip height, $\mathrm{m}$ \\
\hline$I_{1}, \ldots, I_{7}$ & Moment of inertia of the links, kg-m \\
\hline$k$ & Number of particles interact each other \\
\hline$l$ & Hip length, m \\
\hline$l_{1}, l_{2}$ & Distance between the ankle joint and the projection of hip joint, $\mathrm{m}$ \\
\hline$L_{1}, \ldots, L_{7}$ & Link lengths, $\mathrm{m}$ \\
\hline$m_{1}, \ldots, m_{7}$ & Lumped masses of links, $\mathrm{kg}$ \\
\hline$n_{p}$ & Domination count \\
\hline$P_{i}$ & Power consumption, $W$ \\
\hline$P^{\prime}$ & Penalty term \\
\hline Pbest & Particle best solution \\
\hline$q_{1}, \ldots, q_{7}$ & Generalized coordinates (joint angles), degrees \\
\hline$r_{1}, \ldots, r_{7}$ & Distances of lumped masses from the respective joints, $\mathrm{m}$ \\
\hline$s_{h}$ & Height of the staircase, $\mathrm{m}$ \\
\hline$s_{w}$ & Width of the staircase, $\mathrm{m}$ \\
\hline$s_{p}$ & Set of solutions $p$ dominates \\
\hline$t$ & Time interval, s \\
\hline$t_{\text {time }}$ & Time consumed in uniform velocity phase, $\mathrm{s}$ \\
\hline$T$ & Cycle time, $\mathrm{s}$ \\
\hline$v_{i d} i^{t h}$ & particle's updated velocity in $d^{\text {th }}$ dimension \\
\hline$v_{\max }$ & Maximum velocity of the swing leg, $\mathrm{m} / \mathrm{s}$ \\
\hline$V_{i}$ & Velocity vector \\
\hline$V_{\max }$ & Maximum velocity of the robot in one time step, $\mathrm{m} / \mathrm{s}$ \\
\hline$W$ & Width of the supporting foot, $\mathrm{m}$ \\
\hline$W_{\text {in }}$ & Inertia weight \\
\hline$x_{i d}$ & $i^{t h}$ particle's updated position in $d^{t h}$ dimension \\
\hline$x_{i}^{\min }, x_{i}^{\max }$ & Minimum and maximum values of $i^{\text {th }}$ variable \\
\hline$\ddot{x}_{i}$ & Acceleration of link in X-direction, $\mathrm{m} / \mathrm{s}^{2}$ \\
\hline$x_{1}, x_{2}, x_{3}$ & Position of feet placements, $\mathrm{m}$ \\
\hline$x_{D B M}$ & Dynamic balance margin in direction of motion, $\mathrm{m}$ \\
\hline$x_{Z M P}$ & Distance of zero moment point from the ankle joint in direction of motion, $\mathrm{m}$ \\
\hline$X_{i}$ & Particle position vector \\
\hline$\ddot{z}_{i}$ & Acceleration of $i^{t h}$ link in Z-direction, $\mathrm{m} / \mathrm{s}^{2}$ \\
\hline
\end{tabular}


$\delta l \quad$ Small hip movement in $\mathrm{X}$ direction

$\delta h \quad$ Small hip movement in $\mathrm{Z}$ direction

$\mu \quad$ Coefficient of friction

$\omega \quad$ Angular velocity, $\mathrm{rad} / \mathrm{s}$

$\dot{\omega}_{i} \quad$ Angular acceleration of $i^{\text {th }}$ link, $\mathrm{rad} / \mathrm{s}^{2}$

$\tau \quad$ Torque, $\mathrm{N}-\mathrm{m}$

$\theta \quad$ Joint angle, degrees

$\Delta \tau_{i j} \quad$ Change in torque of $i^{\text {th }}$ joint at $j^{\text {th }}$ instant

\section{List of abbreviations}

$\begin{array}{ll}\text { COG } & \text { Centre of gravity } \\ \text { COM } & \text { Centre of mass } \\ \text { COP } & \text { Centre of pressure } \\ \text { DBM } & \text { Dynamic balance margin } \\ \text { DOF } & \text { Degrees of freedom } \\ \text { DSP } & \text { Double support phase } \\ \text { FL } & \text { Fuzzy Logic } \\ \text { GA } & \text { Genetic Algorithm } \\ \text { LIPM } & \text { Linear Inverted pendulum mode } \\ \text { LPM } & \text { Linear Pendulum Mode } \\ \text { PSO } & \text { Particle swarm optimization } \\ \text { MOPSO } & \text { Multi-objective particle swarm optimization } \\ \text { NSGA-II } & \text { Non-dominated sorting genetic algorithm-II } \\ \text { SSP } & \text { Single support phase } \\ \text { ZMP } & \text { Zero Moment Point } \\ \text { rand( ) } & \text { Random number lying in the range of }(0,1) \\ \text { Rand( ) } & \text { Random number lying in the range of }(0,1)\end{array}$

\section{References}

Clerc M and Kennedy J 2002 The particle swarm-explosion, stability and convergence in a multidimensional complex space. IEEE Trans. on Evolutionary Computation 6: 58-78

Das M and Dehuri S 2011 Some studies on particle swarm optimisation for single and multi-objective problems; Integration of Swarm Intelligence and Artificial Neutral Network (Machine Perception and Artificial Intelligence), Dehuri, S., Cho, S.B., Ghosh, S. (eds.), World Scientific Publishing Company; Singapore, First edition, January, pp. 253-254

Deb K, Pratap A, Agarwal S and Meyarivan T 2002 A fast and elitist multiobjective genetic algorithm: NSGA-II. IEEE Trans. Evolutionary Computation 6(2): 182-197

Eberhert R C, Simpson P and Dobbins R 1996 Computational intelligence PC tools: Dalian, San Diego, USA: Academic Press, Ch 6, pp. 212-226

Fu K S, Gonzalez R C and Lee C S G 1987 Robotics: Control, Sensing, Vision, and Intelligence. McGrawHill Inc.

Goldberg D E 1989 Genetic Algorithm in Search Optimization, and Machine Learning, Addison-Wesley, Reading, Massachusetts, USA

Hemami H and Farnsworth R L 1997 Postural and gait stability of a planar five-link biped by simulation. IEEE Trans. Automatic Control 22(3): 452-458 
Holland J H 1975 Adaptation in Natural and Artificial Systems, The University of Michigan Press, Ann Arbor, MI, USA

Ito S and Kawasaki H 2000 A standing posture control based on ground reaction force. Proc. of IEEE/RSJ Intl. Conf. on Intelligent Robotics and Systems, pp 1340-1345

Ito S, Amano S, Sasaki M and Kulvanit P 2008 A ZMP feedback control for biped balance and its application to in-place lateral stepping motion. J. Comput. 3(8): 23-31

Jamshidi N and Rostami M 2008 Gait optimization of biped robot during double support phase by pure dynamic synthesis. Am. J. Appl. Sci. 5(9): 1175-1181

Kennedy J and Eberhart R 1995 Particle swarm optimization. Proc. of IEEE Intl. Conf. on Neural Networks, Perth, Australia, pp. 1942-1948

Li Q, Takanishi A and Kato I 1991 A biped walking robot having a ZMP measurement system using universal force-moment sensors, Proc. of IEEE/RSJ Intl. Workshop on Intelligent Robots and Systems IROS '91. Nov. 3-5, Osaka, Japan, pp. 1568-1573

Lin J, Chang J, Lyu S M, Wang S W and Lin Y W 2010 Locomotion control of a biped robot for stair-climbing by fuzzy stabilization tuning approach. Proc. of IEEE Multi-Conference on Systems and Control. Yokohama, Japan, September 8-10, pp. 1590-1595

Mitobe K, Mori N and Nasu Y 1997 Control of a biped walking robot during the double support phase. Autonomous Robots 4: 287-296

Nishii J, Ogawa K and Suzuki R 1998 The Optimal gait pattern in hexapods based on energetic efficiency, Proc. of $3^{\text {rd }}$ Intl Symposium on Artificial life and Robotics, 1, pp. 106-109

Pratihar D K 2008 Soft Computing, Narosa Publishing House, New Delhi, India

Raquel C R and Naval P C Jr 2005 An effective use of crowding distance in multi-objective particle swarm optimization. Proc. of Genetic and Evolutionary Computing Conf. 2005(GECCO '05), pp. 257-264, June 25-29, Washington DC, USA

Sardain P and Bessonnet G 2004 Forces acting on a biped robot. Centre of Pressure-Zero Moment Point. IEEE Trans. on Systems, Man and Cybernetics-Part A: Systems and Humans 34(5): 630-637

Shi Y and Eberhart R C 1999 Empirical study of particle swarm optimization. Proc. of IEEE Intl. Congress on Evolutionary Computation, 3, Washington DC, USA, 101-106

Vukobratovic M, Frank A A and Juricic D 1970 On the stability of biped locomotion. IEEE Trans. Biomedical Eng. 17(1): 25-36

Vundavilli P R and Pratihar D K 2010 Dynamically balanced optimal gaits of a ditch-crossing biped robot. Robotics and Autonomous Syst 58: 349-361

Vundavilli P R and Pratihar D K 2011a Balanced gait generations of a two-legged robot on sloping surface. Sadhana 36(4): 525-550

Vundavilli P R and Pratihar D K 2011b Near-optimal gait generations of a two-legged robot on rough terrains using soft computing. Robotics and Computer-Integrated Manufacturing 27: 521-530

Vundavilli P R, Sahu S K and Pratihar D K 2007a Dynamically balanced ascending and descending gaits of a two-legged robot. Int. J. Humanoid Robotics 4(4): 717-751

Vundavilli P R, Sahu S K and Pratihar D K 2007b Online dynamically balanced ascending and descending gait generation of a biped robot using soft computing. Int. J. Humanoid Robotics 4(4): 777-814

Wieber P B 2002 On the stability of walking systems. Proc. of Intl. Workshop on Humanoid and Human Friendly Robotics. Tsukuba, Japan, pp 53-59

www.particleswarm.info, accessed from January to July 2010 